\title{
STUDY OF THE MAGNETIC FIELD OF THE GALAXY \\ USING CORRELATION FUNCTIONS OF THE INTENSITY \\ OF SYNCHROTRON BACKGROUND RADIO EMISSION
}

\author{
V.R. SHOUTENKOV \\ Radioastronomy Station, Astro Space Center of \\ Lebedev Physical Institute, Moscow, Russia
}

The possibility to study magnetic field of the Galaxy calculating correlation or structure functions of synchrotron background radio emission have been known long ago (Kaplan and Pikel'ner (1963); Getmantsev (1958)). But this method had not been as popular as other methods of magnetic field studies. However theoretical calculations made by Chibisov and Ptuskin (1981) showed that correlation functions of intensity of synchrotron background radio emission can give a lot of valuable information about galactic magnetic fields because of the intensity of synchrotron background radio emission depends on $H_{\perp}$. According to this theory correlation $C(\theta, \varphi)$ and structure $S(\theta, \varphi)$ functions of intensity, as functions of angular separation $\theta$ between two lines of sight and position angle $\varphi$ on the sky between this two lines of sight, can be presented as a sum of isotropic (not dependent from angle $\varphi$ ) and anisotropic parts:

$$
\begin{aligned}
& C(\theta, \varphi)=C_{i}(\theta)+C_{a}(\theta) \cos (2 \varphi) \\
& S(\theta, \varphi)=S_{i}(\theta)+S_{a}(\theta) \cos (2 \varphi) .
\end{aligned}
$$

The anisotropy of correlation or structure functions is determined by the presence of regular component of magnetic field. Later Chibisov and Lazarian (1987) made other theoretical calculations and have essentially completed this method. They have shown the possibility to find correlation functions of random magnetic field from the amplitude of anisotropic part of correlation function $C_{a}(\theta)$. First observations of intensity of background radio emission have been made with Large Phased Array of Lebedev Physical Institute at $102.5 \mathrm{MHz}$ and the results of analysis of correlation function of intensity have been published by Dagkesamanskii and Shoutenkov (1987) and Lazarian and Shoutenkov (1990). In this short publication I should 


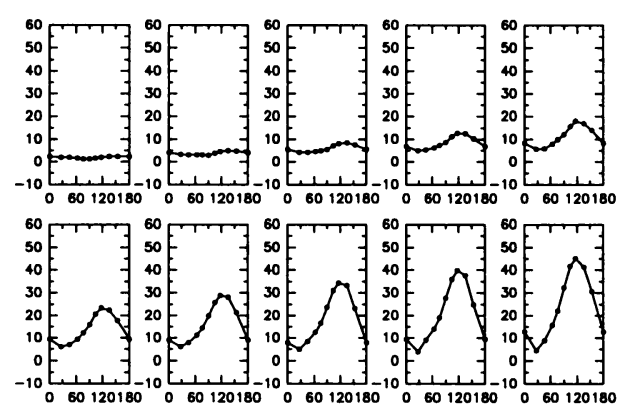

Figure 1. Structure function of intensity $\left(J y^{2}\right)$ plotted versus position angle $\varphi$ for ten values of separation angle $\theta\left({ }^{\circ}\right)$.

like to present new observational data and the results of calculations of the structure function of intensity.

The observations have been made with Large Phased Array at 102.5 $\mathrm{MHz}$ as part of Northern sky survey. The studied area has coordinates from $8^{h} 00^{m}$ to $17^{h} 00^{m}$ in right ascension and from $31^{\circ}$ to $51^{\circ}$ in declination. After data reduction, calibration and subtraction of discrete radio sources the contour map of intensity at $102.5 \mathrm{MHz}$ have been obtained for this area. The map of this large area will be presented elsewhere.

The studied area was divided into nine smaller overlaped regions about $2^{h}$ wide. And for all these regions correlation and structure functions of intensity of background radio emission were calculated. Here I shall discuss only structure functions of background intensity. Structure function of intensity for the first region with right ascension from $8^{h}$ to $10^{h}$ is presented at Fig.1.

The cosine dependence of structure function is clearly seen on this figure. The phase of cosine determines the position angle of regular magnetic field direction in this region. The structure functions in different regions show some changes of the cosine phases and it means changes in direction of regular magnetic field. The difference between the directions of regular magnetic field for the first and last regions is about $50^{\circ}$.

\section{References}

Kaplan, S.A.,Pikel'ner, S.B. (1963) The interstellar medium. Phys. Math. Press, Moscow. Getmantsev, G.G. (1958) Izvestia VUZ. Radiofizica, 1, p. 158.

Chibisov, G.V., Ptuskin, V.S. (1981) 17th Int. Cosmic Ray Conf., Paris, 2, p. 233.

Chibisov, G.V., Lazarian, A.L. (1987) Preprint 283, FIAN, Moscow.

Dagkesamanskii, R.D., Shoutenkov, V.R. (1987) Sov. Astron. Lett., Vol. 13, no. 2, pp. 7376.

Lazarian, A.L, Shoutenkov, V.R. Sov. Astron. Lett., Vol. 16, no. 4, pp. 297-300. 